\title{
Removal of trace organics from aqueous solutions. Effect of membrane thickness*
}

\author{
H.H. Nijhuis**, M.H.V. Mulder and C.A. Smolders \\ University of Twente, Department of Chemical Technology, P.O. Box 217, 7500 AE Enschede \\ (Netherlands)
}

(Received October 29, 1989)

\begin{abstract}
A resistance-in-series model is used to describe the pervaporation performance of elastomeric membranes in the removal of volatile organic components from water. Equations have been derived to describe the organic component flux as a function of feed concentration, permeability of the organic component in the membrane, membrane thickness and liquid boundary layer mass transfer coefficient. The model has been verified using both homogeneous and composite membranes of polydimethylsiloxane, ethylene propylene rubber and polyoctenamer. Membranes with a wide range of thicknesses have been prepared and the pervaporation behaviour for the removal of toluene and trichloroethylene from aqueous solutions has been studied. The experiments show that the hydrodynamic boundary layer resistance is of great importance. For highly permeable polymers such as polydimethylsiloxane mass transfer in the boundary layer is rate determining and should be considered carefully in further development of the process. For less permeable polymers such as ethylene propylene rubber this effect becomes more dominant with decreasing membrane thickness. The water fluxes are inversely proportional to the thickness of the actual separating layer and they depend strongly on the type of elastomer used. A proper choice of the elastomeric material and the thickness of the separating layer will determine the selectivity of the process.
\end{abstract}

Keywords: pervaporation; theory; volatile organics, removal of; polydimethylsiloxane; ethylene propylene rubber; polyoctenamer

\section{Introduction}

Pervaporation offers good prospects in the removal of trace organics from aqueous solutions. The attractiveness of pervaporation in contrast to existing techniques lies in the fact that the pollutant is selectively removed from the feed as an almost pure liquid. Besides the use of pervaporation in ground water

\footnotetext{
*Paper presented at the 6th International Symposium on "Synthetic Membranes in Science and Industry", Tübingen, September 4-8, 1989.

**To whom correspondence should be addressed. Present address: ATO-DLO, Agrotechnological Research Institute, P.O. Rnx 17, 6700 AA Wageningen (The Netherlands).
} 
treatment, other interesting applications are the off shore treatment of aqueous waste streams and the integration in existing industrial processes.

In a previous study [1] extremely high selectivities are reported for relatively thick homogeneous membranes. A decrease in membrane thickness should further improve the already reasonable fluxes. Several authors, however, pointed out the important role of a hydrodynamic boundary layer at the liquid/membrane interface [2-5] causing a strong decrease of pervaporation selectivity using thinner membranes. In this work the effect of membrane thickness on the performance of pervaporation membranes in the removal of volatile organic components from water has been studied.

\section{Theory}

\subsection{Overall mass transfer resistance}

Psaume et al. [2] were probably one of the first to recognize and quantitatively describe the problem of concentration polarization in the pervaporation of trace organics from aqueous solutions. They developed a physical model based on the film theory neglecting any mass transfer resistance of the membrane itself. Côté and Lipski [3] showed by model calculations, however, that membrane resistance cannot always be neglected. They discussed a resistancein-series model to describe the pervaporation performance of a silicone rubber hollow fiber module.

From a mass balance in a volume element between $x=0$ and $x=x_{1}$ at the feed side of the membrane in Fig. 1, equations can be derived to describe the transport of the preferentially permeating component 1 in the liquid boundary layer. The flux can simply be described as [3]:

$J_{1}=k_{\mathrm{OV}} \phi_{1}^{\mathrm{b}}$

From measurements of the feed concentration and the steady state organic

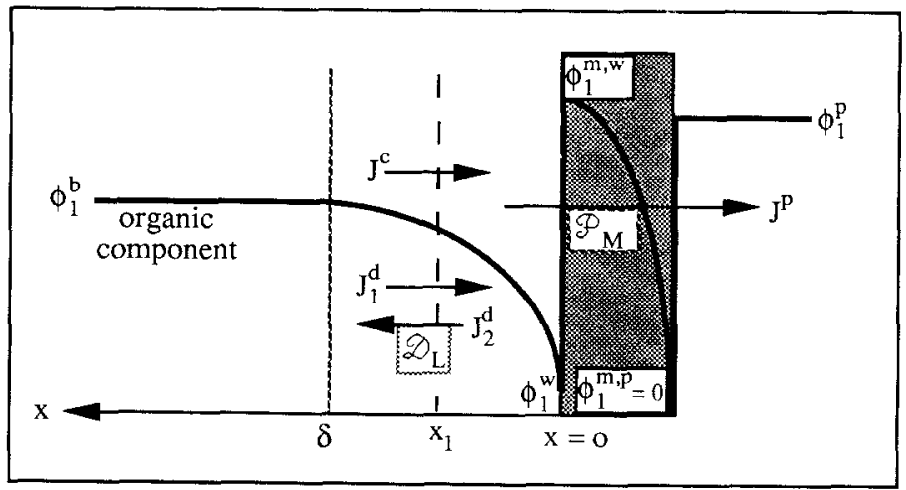

Fig. 1. Concentration profile for the preferentially permeating component 1. 
component flux in the pervaporation experiments the so called overall mass transfer coefficient $\left(k_{\mathrm{OV}}\right)$ can be determined.

If composite membranes are used with a negligible resistance of the porous support to mass transfer and under the strict condition that the pressure at the permeate side approaches zero the overall mass transfer resistance $\left(1 / k_{\mathrm{OV}}\right)$ consists of the sum of membrane resistance $\left(1 / k_{\mathrm{M}}\right)$ and liquid boundary layer resistance $\left(1 / k_{\mathrm{L}}\right)$.

$\frac{1}{k_{\mathrm{OV}}}=\frac{1}{k_{\mathrm{L}}}+\frac{1}{k_{\mathrm{M}}}=\frac{1}{k_{\mathrm{L}}}+\frac{\ell}{\mathscr{P}}$

From eqns. (1) and (2) it can be seen that the pervaporation performance in the removal of volatile organic components from water is influenced by the following parameters:

- feed concentration $\left(\phi_{1}^{\mathrm{b}}\right)$,

- liquid boundary layer mass transfer coefficient $\left(k_{1}\right)$,

- membrane permeability $(\mathscr{P})$, and

- membrane thickness $(\ell)$.

\subsection{Determination of the liquid boundary layer and membrane mass transfer resistance}

The liquid boundary layer resistance $\left(1 / k_{\mathrm{L}}\right)$ is strongly dependent on the flow conditions at the feed side of the membrane. The membrane resistance is related to parameters like membrane thickness and permeability. With increasing cross-flow velocity (increasing Reynolds number) the boundary layer resistance decreases and the membrane resistance $\left(1 / k_{\mathrm{M}}\right)$ becomes more important. Especially for relatively thick homogeneous membranes with a low permeability for the organic component the membrane resistance, which is supposed to be directly proportional to the membrane thickness, will be rate determining. By plotting the overall mass transfer resistance as a function of the membrane thickness a straight line is obtained for which the intercept is equal to the liquid boundary layer resistance and the slope is equal to the reciprocal value of the membrane permeability (see Fig. 2).

\section{Experimental}

\subsection{Materials and membrane preparation}

Three polymer materials have been selected on the basis of a previous study [1]. Silicone rubber (PDMS) is selected, because of its extremely high permeation rates for several penetrants. Ethylene propylene rubber (EPDM) and polyoctenamer $\left(\mathrm{OT} ;-\left(\mathrm{CH}_{2}\right)_{3} \mathrm{CH}=\mathrm{CH}\left(\mathrm{CH}_{2}\right)_{3}{ }^{-}\right)$have been investigated because they combine extremely high selectivities towards the organic component with good organic component fluxes. A standard casting procedure was 


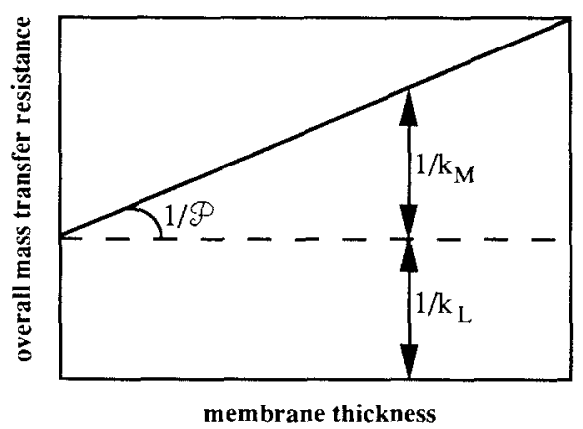

Fig. 2. Determination of the liquid boundary layer and the membrane resistance.

followed to prepare peroxide cured dense homogeneous and multilayer composite membranes.

\subsection{Membrane characterization}

The porous substructure was characterized by measurement of the pure water flux at a pressure of 3 bar with a standard laboratory ultrafiltration set-up.

The thickness of the applied PDMS toplayers was determined by measurement of oxygen and nitrogen permeability. Provided that the gas permeability of PDMS is known the effective membrane thickness can be calculated from this value assuming an inverse proportionality. Furthermore the membrane area should be known, wheres the pressure difference over the membrane and the gas permeate flow should be determined. A permeability ratio for oxygen/ nitrogen of at least two indicates that the membrane is defect free. The permeation rates for EPDM and OT were too small to be mcasurcd in our simple gas permeation set-up. Their toplayer thickness was therefore determined in another way as will be described below.

\subsection{Pervaporation experiments}

The experimental pervaporation data have been obtained with a standard laboratory set-up consisting of a $5 \mathrm{l}$ thermo-regulated storage tank with Viton ${ }^{(1)}$ feed and recycle lines. The concentration in the feed tank is kept constant by a continuous supply of the organic component. For all experiments the feed is recycled with a centrifugal pump at a constant cross-flow velocity. An average flow rate of approximately $2.0 \mathrm{l} / \mathrm{min}$ is large enough to prevent a change in concentration over four pervaporation cells in series with an effective membrane area of $75 \mathrm{~cm}^{2}$. The pervaporation cell schematically represented in Fig. 3 consists of an upstream and a downstream half cell. The liquid feed flows through the upstream side bringing the upper side of the membrane in contact with the liquid. The pressure at the permeate side was kept below 3 mbar by a vacuum pump. 


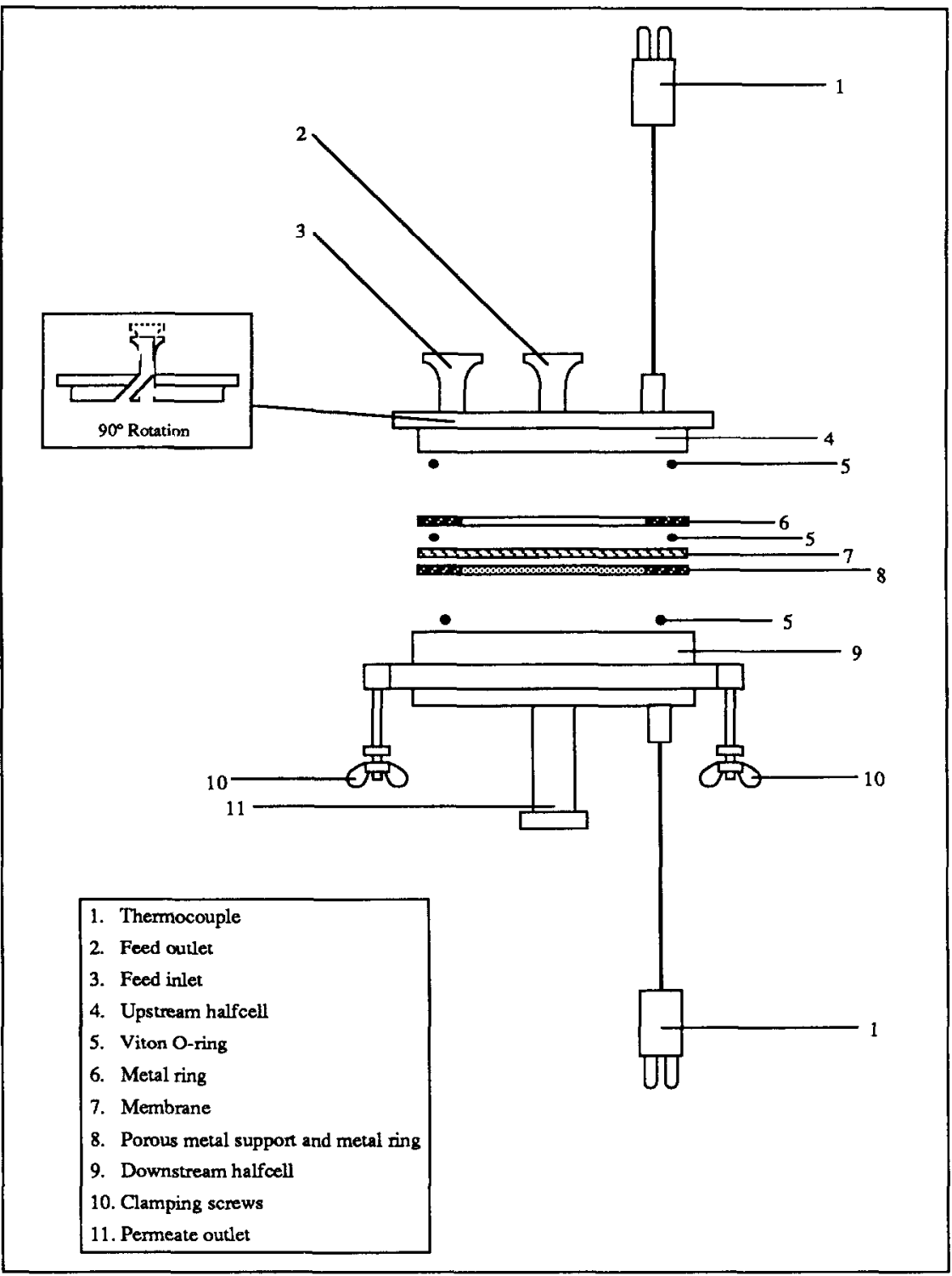

Fig. 3. Schematical representation of the pervaporation cell.

After steady state conditions had been achieved the permeate was collected in cold traps cooled with liquid nitrogen. The volume component flux $J_{i}$ can easily be calculated according to the following equation:

component flux: $J_{i} \rho_{i}=\frac{m}{A t} w_{i}^{\text {p }}$ 
The concentrations of the liquid mixtures were in the range of $100-500 \mu \mathrm{g} /$ $\mathrm{g}$ which allows to assume a concentration independent feed density of approximately $1000 \mathrm{~kg} / \mathrm{m}^{3}$.

\section{Results and discussion}

\subsection{Support}

Ultrafiltration type of polyvinylidenefluoride (PVDF) and polysulfone (PSf) membranes obtained by immersion precipitation have been used as support for the elastomeric toplayer. The resistance of the porous support was determined from pervaporation experiments. In Fig. 4 the flux of the organic component is given as a function of the feed concentration for a homogeneous OTmembrane. The same membrane is used a second time, but now in combination with a porous support. The organic component flux is not altered which shows that the PSf-support has negligible resistance towards the organic component under the pervaporation conditions applied in this study.

\subsection{Permeation of water}

The water fluxes which are independent of the concentration of organics in the aqueous solution [6-8] appear to be inversely proportional to the membrane thickness both for composite and homogeneous membranes of PDMS and for homogeneous membranes of EPDM (see Fig. 5). It can be seen that a large difference in the water fluxes is observed for the two polymer materials used.

'Toplayer thicknesses of composite membranes can now be determined by mcasuring the pure water flux. From the ratio of this flux and the pure water flux of a homogeneous membrane with a known thickness the effective thickness of the composite membrane can be calculated. Electron microscopy can be used as an additional control. SEM, however, gives a local toplayer thick-

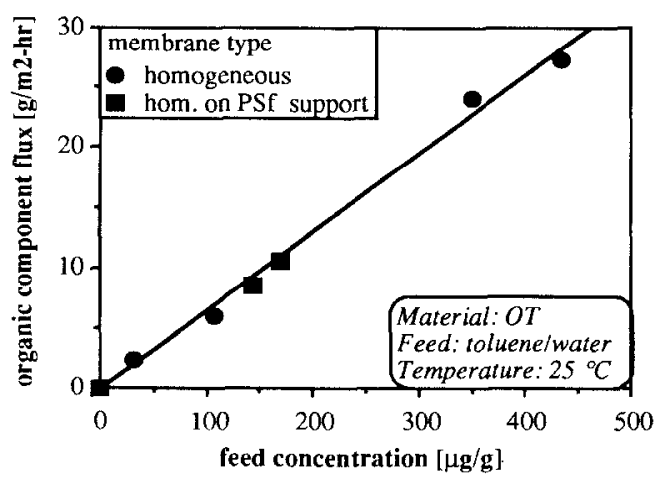

Fig. 4. Influence of porous support on pervaporation performance. 

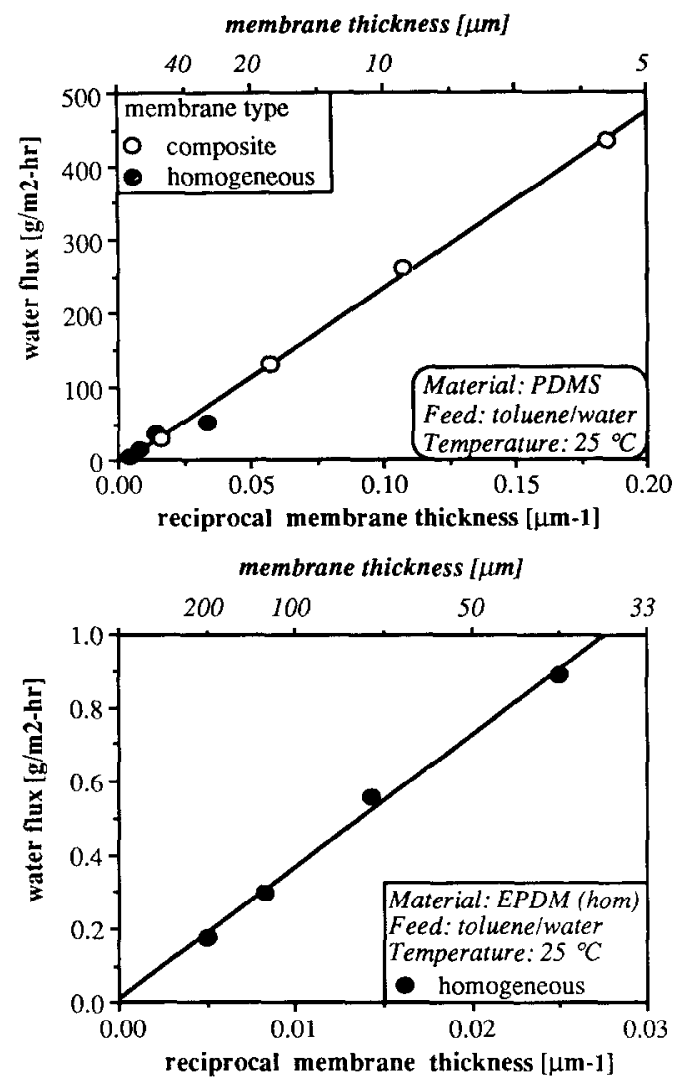

Fig. 5. Water fluxes obtained from liquid feed mixtures as a function of reciprocal membrane thickness for PDMS (top) and EPDM (bottom) membranes.

ness in contrast to the overall thickness determined from the permeability measurements.

\subsection{Determination of the overall mass transfer coefficient}

The overall mass transfer coefficient can be determined from the organic component flux as a function of the feed concentration. According to eqn. (1) a straight line through the origin should be obtained with a slope equal to the mass transfer coefficient. Figure 6 shows the trichloroethylene flux as a function of the feed concentration for two homogeneous EPDM membrane of which the thicknesses differ by a factor of 3 . The mass transfer coefficients calculated from these curves are $0.9 \times 10^{-5} \mathrm{~m} / \mathrm{sec}$ for the thicker and $1.3 \times 10^{-5} \mathrm{~m} / \mathrm{sec}$ for the thinner membrane. These results indicate that an inverse proportionality between organic component flux and membrane thickness could not be established. This observation justifies the conclusion that besides a membrane resistance an additional resistance, originating from the liquid boundary layer 


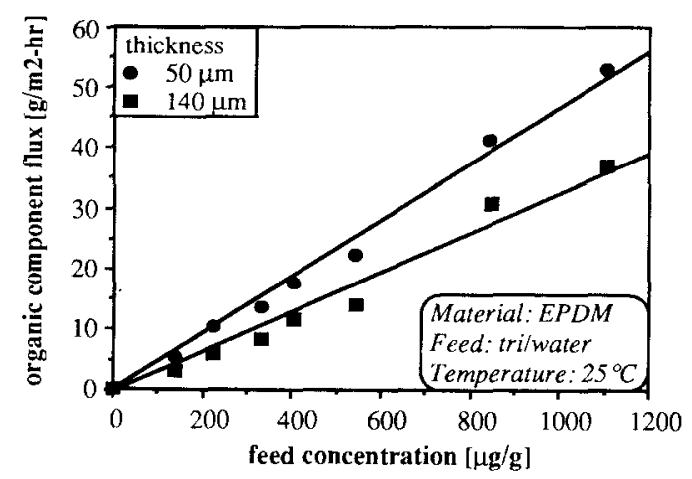

Fig. 6. Organic component flux vs concentration for EPDM membranes.

at the membrane interface, contributes to the pervaporation performance in the removal of trace organics from aqueous solutions. In the following section the contributions of both the liquid boundary layer and the membrane to the total resistance against transport of the organic component will be determined.

\subsection{Determination of mass transfer resistances}

The overall mass transfer coefficient $\left(k_{\mathrm{OV}}\right)$ can be calculated from a plot of the organic component flux vs. the concentration (see Fig. 6). Because straight lines are obtained an alternative, less time consuming, way of determining the overall resistance has been used. The ratio of the organic component flux and its bulk concentration at one fixed composition of the feed mixture gives the desired value for the overall mass transfer coefficient. For dense homogeneous membranes of PDMS and EPDM the boundary layer and the membrane resistance have been determined in this way from experiments with toluene as the organic component.

The component fluxes for dense homogeneous membranes of PDMS and EPDM with thicknesses in the range of $30-240 \mu \mathrm{m}$ are given in Fig. 7. Organic component fluxes of $13-20 \mathrm{~g} /\left(\mathrm{m}^{2}-\mathrm{hr}\right)$ at a constant and fixed concentration of $250 \mu \mathrm{g} / \mathrm{g}$ are obtained for PDMS membranes, while the water fluxes range from $6-51 \mathrm{~g} /\left(\mathrm{m}^{2}-\mathrm{hr}\right)$. For EPDM membranes toluene fluxes at a constant concentration of $250 \mu \mathrm{g} / \mathrm{g}$ could be calculated of $3.5-8.5 \mathrm{~g} /\left(\mathrm{m}^{2}-\mathrm{hr}\right)$ while the water fluxes range from $0.17-0.89 \mathrm{~g} /\left(\mathrm{m}^{2}-\mathrm{hr}\right)$.

Figure 8 gives the calculated overall resistance $\left(1 / k_{\mathrm{OV}}\right)$ as a function of the membrane thickness for PDMS and EPDM membranes in the separation of toluene/water mixtures. For comparison the results with trichloroethylene/ water mixtures for both homogeneous and composite PDMS membranes as well as homogeneous EPDM membranes are also presented in these figures.

The data clearly indicate that the permeabilities obtained from the reciprocal slopes of the lines after linear regression are higher in PDMS than in 

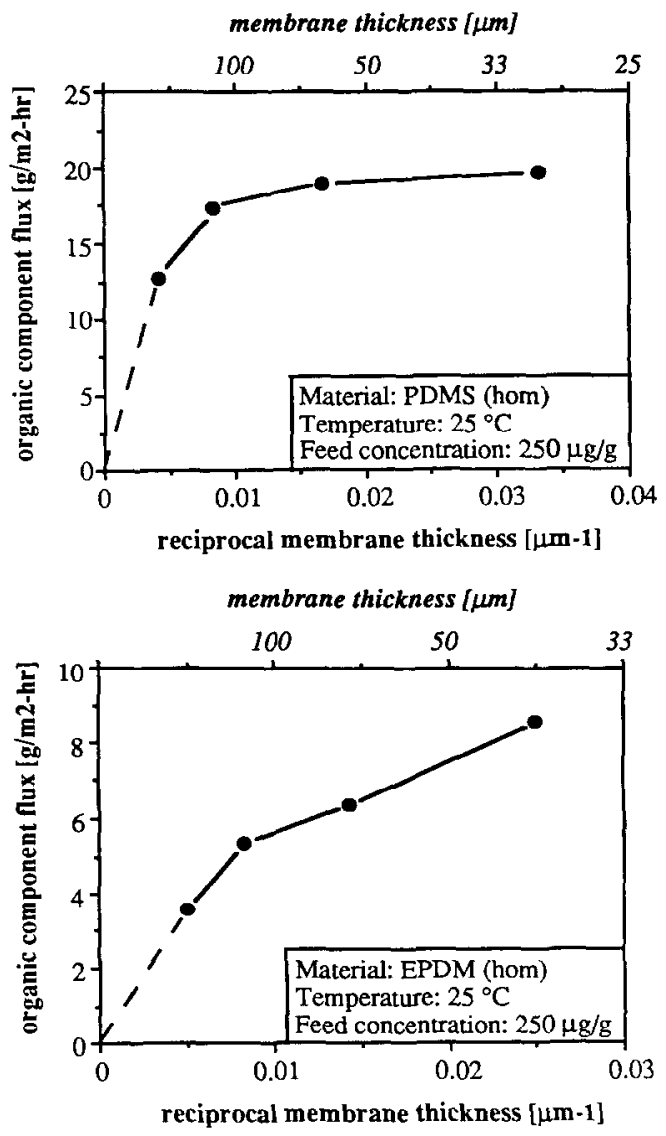

Fig. 7. Organic component fluxes as a function of reciprocal membrane thickness for PDMS (top) and EPDM (bottom) membranes.

EPDM especially for toluene. This is in conformation with results presented earlier [1]. The determination of the trichloroethylene permeability in PDMS is difficult due to the small variations in organic component fluxes and the relatively small range of thicknesses investigated. The regression curve in Fig. 8 is drawn on the basis of the results with composite membranes and one homogeneous membrane. The overall resistance determined for the $17.5 \mu \mathrm{m}$ membrane was almost identical to the values obtained for the $72 \mu \mathrm{m}$ composite and the $80 \mu \mathrm{m}$ homogenous membrane. Therefore this value was left out of the fitting procedure.

Table 1 summarizes the results obtained for the various systems. This table also contains the results of experiments for the system trichloroethylene/water/ OT (composite on PVDF) in which the thicknesses of the selective layer were ca. 5 and $150 \mu \mathrm{m}$.

The intercepts of the curves, expressing the liquid boundary layer resis- 

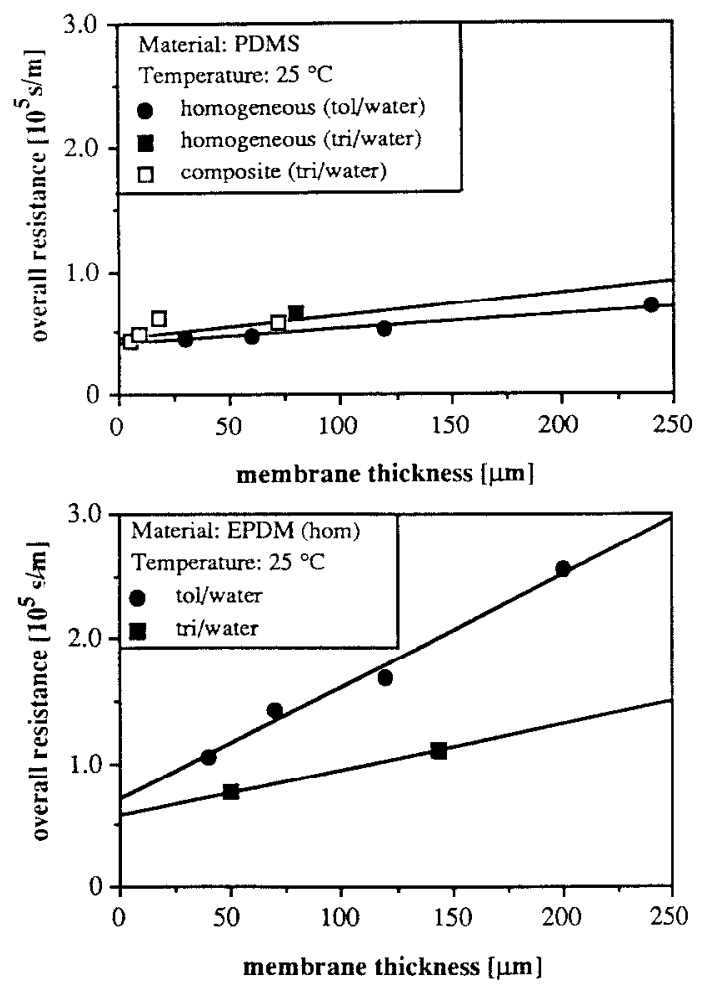

Fig. 8. Mass transfer resistances as a function of membrane thickness for PDMS (top) and EPDM (bottom) separating layers.

TABLE 1

Mass transfer coefficient for elastomeric pervaporation membranes

\begin{tabular}{llll}
\hline Material & $\begin{array}{l}\text { Organic } \\
\text { component }\end{array}$ & \multicolumn{2}{l}{ Mass transfer coefficient $(\mathrm{m} / \mathrm{sec})$} \\
\cline { 3 - 4 } & & Liquid film & Membrane $(100 \mu \mathrm{m})$ \\
\hline PDMS & toluene & $2.5 \times 10^{-5}$ & $8.2 \times 10^{-5}$ \\
PDMS (Comp) & tri & $2.3 \times 10^{-5}$ & $5.4 \times 10^{-5}$ \\
EPDM & toluene & $1.4 \times 10^{-5}$ & $1.1 \times 10^{-5}$ \\
EPDM & tri & $1.7 \times 10^{-5}$ & $2.8 \times 10^{-5}$ \\
OT (Comp) & tri & $1.7 \times 10^{-5}$ & $2.8 \times 10^{-5}$ \\
\hline
\end{tabular}

tances $\left(1 / k_{\mathrm{L}}\right)$, are calculated by linear regression and give values of the mass transfer coefficient in the range of $1.4-2.5 \times 10^{-5} \mathrm{~m} / \mathrm{sec}$. In principle the liquid boundary layer resistances should be independent of the type of polymer material used. The observed deviations are probably caused by the extrapolation procedure used. The average liquid boundary layer mass transfer coefficient 
calculated from the above values is $1.9( \pm 0.5) \times 10^{-5} \mathrm{~m} / \mathrm{sec}$, which is a pretty high, but not an unrealistic; value to be reached in practical applications.

The membrane mass transfer coefficient is dependent of the membrane thickness, but for a $10 \mu \mathrm{m}$ PDMS membrane this coefficient $\left(k_{\mathrm{M}}\right)$ takes a value of $8.2 \times 10^{-4} \mathrm{~m} / \mathrm{sec}$ for toluene. This indicates that for such a membrane the organic component flux is determined predominantly by the liquid boundary layer resistance and not by the choice of the elastomeric material. Especially for PDMS the contribution of the membrane resistance can almost be neglected. In the case of the more selective EPDM membranes the membrane resistance might become more important.

In this work the attention was focussed on the determination of mass transfer coefficients in the liquid boundary layer and in the membrane respectively from thickness dependent pervaporation measurements. In a forthcoming paper the results will be discussed of experiments with the boundary layer mass transfer coefficient as the most important variable [9].

\section{Conclusions}

A resistance-in-series model can be used to determine the mass transfer coefficients in the removal of volatile organic components from water by a pervaporation process.

Under the conditions applied the resistance of the porous supports used for the preparation of composite membranes was negligible although no optimization was performed. In this study it is shown that the resistance of the liquid boundary layer can not be neglected in the removal of trace organics from aqueous solutions. The flux of the organic component through the membrane is mainly determined by the liquid boundary layer resistance against mass transfer and only to a minor extent by the choice of the elastomeric material. For elastomers with a lower permeability, such as EPDM, the membrane resistance may become more important. The water flux appears to be inversely proportional to the thickness of the separating layer and it depends strongly on the type of elastomer used. Choosing the elastomer with the highest selectivity seems obvious.

\section{List of symbols}

A membrane area $\left(\mathrm{m}^{2}\right)$

$\mathscr{D}$ diffusion coefficient $\left(\mathrm{m}^{2} / \mathrm{sec}\right)$

$J$ pervaporation volume flux $\left[\mathrm{m}^{3} /\left(\mathrm{m}_{2}-\mathrm{sec}\right)\right]$

$k$ mass transfer coefficient $(\mathrm{m} / \mathrm{sec})$

$l$ membrane thickness (m)

$m \quad$ weight of collected permeate $(\mathrm{kg})$

$\mathscr{P}$ permeability in pervaporation experiments $\left(\mathrm{m}^{2} / \mathrm{sec}\right)$ 
$t \quad$ permeation time $(\mathrm{sec})$

$w \quad$ weight fraction $(\mathrm{kg} / \mathrm{kg})$

$x \quad$ distance from the membrane wall $(\mathrm{m})$

\title{
Greek symbols
}

$\delta \quad$ thickness of the liquid boundary layer (m)

$\phi \quad$ volume fraction $\left(\mathrm{m}^{3} / \mathrm{m}^{3}\right)$

$\rho \quad$ liquid density $\left(\mathrm{kg} / \mathrm{m}^{3}\right)$

\author{
Subscripts \\ 1 preferentially permeating component \\ 2 less permeable component \\ $i \quad$ component $i$ \\ $\mathrm{L} \quad$ liquid boundary layer \\ M membrane \\ OV overall
}

\section{Superscripts}

b bulk feed solution

c convection

d diffusion

$\mathrm{m}, \mathrm{p}$ interface membrane/permeate (in the membrane)

$\mathrm{m}, \mathrm{w}$ interface membrane/feed (in the membrane)

$\mathrm{p}$ permeate

\section{References}

1 H.H. Nijhuis, M.H.V. Mulder and C.A. Smolders, Selection of elastomeric membranes for the removal of volatile organic components from water, Proc. 3rd Int. Conf. on Perv. Processes in the Chem. Ind., Nancy (France), R. Bakish (Ed.), Bakish Materials Corporation, Englewood, NJ, 1988, p. 239.

2 R. Psaume, Ph. Aptel, Y. Aurelle, J.C. Mora and J.L. Bersillon, Pervaporation: Importance of concentration polarization in the extraction of trace organics from water, J. Membrane Sci., 36 (1988) 373-384.

3 P. Côté and C. Lipski, Mass transfer limitations in pervaporation of water and waste water treatment, Proc. 3rd Int. Conf. on Perv. Prucesses in the Chem. Ind., Nancy (France), R. Bakish (Ed.), Bakish Materials Corporation, Englewood, NJ, 1988, p. 449.

4 J.W.F. Spitzen, Pervaporation (membranes and models for the dehydration of ethanol), Ph.D. Thesis, University of Twente, Fnschede, The Netherlands, 1988.

5 H.C.W.M. Buys, H.F. Martens, J.W. van Heuven, A.E. Jansen and A.J.A. Tinnemans, New intrinsic separation characteristics of poly (dimethylsiloxane) membranes for organic vapour/ nitrogen gas mixtures, Membraantechnologie, Vol. 4, Stam, 1989, p. 39.

6 T.Q. Nguyen and K. Nobe, Extraction of organic contaminants in aqueous solutions by pervaporation, J. Membrane Sci., 30 (1987) 11-22.

7 K.W. Böddeker, Pervaporation durch Membranen und ihre Anwendung zur Trennung von Flüssiggemischen, VDI Verlag GmbH, Düsseldorf, Germany, 1986. 
8 J.P. Brun, C. Larchet, G. Bulvestre and B. Auclair, Sorption and pervaporation of dilute aqueous solutions of organic compounds through polymer membranes, J. Membrane Sci., 25 (1985) 35-100.

9 H.H. Nijhuis, M.H.V. Mulder and C.A. Smolders, Removal of trace organics from water by pervaporation; Boundary layer effects, to he published. 